\title{
Quantitative Thermo-acoustics and related problems
}

\author{
Guillaume Bal \\ Department of Applied Physics \& Applied Mathematics, Columbia University, New \\ York, NY 10027 \\ E-mail: gb2030@columbia.edu
}

\section{Kui Ren}

Department of Mathematics, University of Texas at Austin, Austin, TX 78712

E-mail: ren@math.utexas.edu

\section{Gunther Uhlmann}

Department of Mathematics, UC Irvine, Irvine, CA 92697 and Department of Mathematics, University of Washington, Seattle, WA 98195

E-mail: gunther@math . washington.edu

\section{Ting Zhou}

Department of Mathematics, University of Washington, Seattle, WA 98195

E-mail: tzhou@math.washington.edu

\begin{abstract}
Thermo-acoustic tomography is a hybrid medical imaging modality that aims to combine the good optical contrast observed in tissues with the good resolution properties of ultrasounds. Thermo-acoustic imaging may be decomposed into two steps. The first step aims at reconstructing an amount of electromagnetic radiation absorbed by tissues from boundary measurements of ultrasounds generated by the heating caused by these radiations. We assume this first step done. Quantitative thermo-acoustics then consists of reconstructing the conductivity coefficient in the equation modeling radiation from the now known absorbed radiation. This second step is the problem of interested in this paper.

Mathematically, quantitative thermo-acoustics consists of reconstructing the conductivity in Maxwell's equations from available internal data that are linear in the conductivity and quadratic in the electric field. We consider several inverse problems of this type with applications in thermo-acoustics as well as in acousto-optics. In this framework, we obtain uniqueness and stability results under a smallness constraint on the conductivity. This smallness constraint is removed in the specific of a scalar model for electromagnetic wave propagation for appropriate illuminations constructed by the method of complex geometric optics (CGO) solutions.
\end{abstract}

Keywords. Photo-acoustics, Thermo-acoustics, inverse scattering with internal data, inverse problems, acousto-optics stability estimates, complex geometrical optics (CGO) solutions. 


\section{Introduction}

Thermo-acoustic tomography, and more generally photo-acoustic tomography, is based on the photo-acoustic effect: a fraction of propagating radiation is absorbed by the underlying medium. This results in local heating and hence local mechanical expansion of the medium. This in turn generates acoustic pulses that propagate to the domain's boundary. The acoustic signal is measured at the domain's boundary and used to reconstruct the amount of absorbed radiation. The reconstruction of absorbed radiation is the first step in both thermo-acoustic and photo-acoustic tomography. What distinguishes the two modalities is that in photo-acoustic tomography, radiation is highfrequency radiation (near-infra-red with sub- $\mu m$ wavelength) while in thermo-acoustic, radiation is low-frequency radiation (microwave with wavelengths comparable to $1 \mathrm{~m}$ ).

The second step in photo- and thermo-acoustic tomography is called quantitative photo- or thermo-acoustics and consists of reconstructing the absorption coefficient from knowledge of the amount of absorbed radiation. This second step is different in thermoacoustic and photo-acoustics as radiation is typically modeled by Maxwell's equations in the former case and transport or diffusion equations in the latter case. The problem of interest in the paper is quantitative thermo-acoustics.

For physical descriptions of the photo-acoustic and thermo-acoustic effects, we refer the reader to the works $[5,6,7,11,25,26]$ and their references. For the mathematical aspects of the first step in thermo- and photo-acoustics, namely the reconstruction of the absorbed radiation map from boundary acoustic wave measurements, we refer the reader to e.g. $[1,9,10,12,13,14,17,19,22]$. Serious difficulties may need to be addressed in this first step, such as e.g. limited data, spatially varying acoustic sound speed $[1,14,22]$, and the effects of acoustic wave attenuation [16]. In this paper, we assume that the absorbed radiation map has been reconstructed satisfactorily.

In thermo-acoustics, where radiation is modeled by an electromagnetic field $E(x)$ solving (time-harmonic) Maxwell's equations, absorbed radiation is described by the product $H(x)=\sigma(x)|E(x)|^{2}$. The second step in the inversion thus concerns the reconstruction of $\sigma(x)$ from knowledge of $H(x)$. This paper presents several uniqueness and stability results showing that in several settings, the reconstruction of $\sigma(x)$ from knowledge of $H(x)$ is a well-posed problem. These good stability properties were observed numerically in e.g., [18], where it is also observed that imaging $H(x)$ may not provide a faithful image for $\sigma(x)$ because of diffraction effects.

In photo-acoustics, radiation is modeled by a radiative transfer equation or a diffusion equation. Several results of uniqueness in the reconstruction of the absorption and scattering coefficients have been obtain recently. We refer the reader to e.g., $[4,6,7,21]$, for works on quantitative photoacoustics in the mathematics and bioengineering literatures.

After recalling the equations modeling radiation propagation and acoustic

propagation, we define in section 2 below the mathematical problems associated to quantitative thermo-acoustics. We mainly consider two problems: a system of time- 
harmonic Maxwell equations and a scalar approximation taking the form of a Helmholtz equation.

This paper presents two different results. The first result, presented in section 3 , is a uniqueness and stability result under the condition that $\sigma$ is sufficiently small (as a bounded function). This result, based on standard energy estimates, works for general problems of the form $\mathcal{H} u=\sigma u$ and includes the thermo-acoustic models both in the vectorial and scalar cases. The same technique also allows us to reconstruct $\sigma$ when $\mathcal{H}=\Delta$ the Laplace operator. This has applications in simplified models of acousto-optics as they arise, e.g., in [3].

The second result removes the smallness constraint on $\sigma$ for the (scalar) Helmholtz model of radiation propagation. The result is based on proving a fixed point iteration for the conductivity $\sigma$ under the assumption that $\sigma$ is sufficiently regular and that the electromagnetic illumination applied to the boundary of the domain is well-chosen. In some sense, the smallness constraint on $\sigma$ is traded against a constraint on the type of electromagnetic illumination that is applied. The method of proof is based on using complex geometric optics (CGO) solutions that are asymptotically, as a parameter $\rho \rightarrow \infty$, independent of the unknown term $\sigma$. Such CGO's allow us to construct a contracting functional on $\sigma$ provided that the electromagnetic illumination is wellchosen (in a non-explicit way). The technique of CGO solutions is similar to their use in quantitative reconstructions in photo-acoustic as they were presented in [4].

\section{Quantitative thermo-acoustics}

\subsection{Modeling of the electromagnetic radiation}

The propagation of electromagnetic radiation is given by the equation for the electric field

$$
\frac{1}{c^{2}} \frac{\partial^{2}}{\partial t^{2}} E+\sigma \mu \frac{\partial}{\partial t} E+\nabla \times \nabla \times E=S(t, x),
$$

for $t \in \mathbb{R}$ and $x \in \mathbb{R}^{3}$. Here, $c^{2}=(\epsilon \mu)^{-1}$ is the light speed in the domain of interest, $\epsilon$ the permittivity, $\mu$ the permeability, and $\sigma=\sigma(x)$ the conductivity we wish to reconstruct.

Let us consider the scalar approximation to the above problem:

$$
\frac{1}{c^{2}} \frac{\partial^{2}}{\partial t^{2}} u+\sigma \mu \frac{\partial}{\partial t} u-\Delta u=S(t, x)
$$

for $t \in \mathbb{R}$ and $x \in \mathbb{R}^{n}$ for $n \geq 2$ an arbitrary dimension. This approximation will be used to simplify some derivations in the paper. One of the main theoretical results in the paper (presented in section 4) is at present obtained only for the simplified scalar setting.

Let $\omega_{c}=c k$ be a given frequency, which we assume corresponds to a wavelength $\lambda=\frac{2 \pi}{k}$ that is comparable or large compared to the domain we wish to image. We assume that $S(t, x)$ is a narrow-band pulse with central frequency $\omega_{c}$ of the form $-e^{-i \omega_{c} t} \phi(t) S(x)$, where $\phi(t)$ is the envelope of the pulse and $S(x)$ is a superposition 
of plane waves with wavenumber $k$ such that $\omega_{c}=c k$. As a consequence, upon taking the Fourier transform in the above equation and assuming that all frequencies satisfy a similar equation, we obtain that

$$
u(t, x) \sim \phi(t) u(x)
$$

where

$$
\Delta u+k^{2} u+i k c \mu \sigma u=S(x)
$$

We recast the above equation as a scattering problem with incoming radiation $u^{i}(x)$ and scattered radiation $u^{s}(x)$ satisfying the proper Sommerfeld radiation conditions at infinity. Replacing $c \mu \sigma$ by $\sigma$ to simplify notation, we thus obtain the model for electromagnetic propagation:

$$
\Delta u+k^{2} u+i k \sigma(x) u=0, \quad u=u^{i}+u^{s}
$$

The incoming radiation $u^{i}$ is a superposition of plane waves of the form $u^{i}=e^{i k \xi \cdot x}$ with $\xi \in \mathbb{S}^{2}$ and is assumed to be controlled experimentally.

\subsection{Modeling of the acoustic radiation}

The amount of absorbed radiation by the underlying medium as the electromagnetic waves propagate is given by

$$
H(t, x)=\sigma(x)|u(t, x)|^{2} \sim \phi^{2}(t) \sigma(x)|u|^{2}(x) .
$$

A thermal expansion (assumed to be proportional to $H$ ) results and acoustic waves are emitted. Such waves are modeled by

$$
\frac{1}{c_{s}^{2}(x)} \frac{\partial^{2} p}{\partial t^{2}}-\Delta p=\beta \frac{\partial}{\partial t} H(t, x)
$$

with $c_{s}$ the sound speed and $\beta$ a coupling coefficient measuring the strength of the photoacoustic effect. We assume $\beta$ to be constant and known. The pressure $p(t, x)$ is then measured on $\partial X$ as a function of time.

The first task in thermo-acoustics is to solve an inverse wave problem consisting of reconstructing $H(t, x)$ from knowledge of $p(t, x)$ measured on $\partial X$ as a function of time. The latter problem is however extremely underdetermined when $H(t, x)$ is an arbitrary function of time and space. In order for the inverse wave problem to be well-posed, restrictions on $H(t, x)$ need to be performed. Typically, a separation of time scales is invoked to ensure that $H(t, x)$ has a support in time that is small compared to the time scale of acoustic wave propagation. When such a separation of scale is valid, we obtain as in [2] that

$$
H(t, x)=H(x) \delta_{0}(t), \quad H(x)=\sigma(x) \int_{\mathbb{R}}|u(t, x)|^{2} d t=\sigma(x)|u(x)|^{2} \int_{\mathbb{R}} \phi^{2}(t) d t .
$$


We assume that the pulse intensity is normalized so that $\int_{\mathbb{R}} \phi^{2}(t) d t=1$. The above separation of scales holds for measurements of acoustic frequencies that are comparable to or smaller than the frequency of the pulse $\phi(t)$. Typical experiments are performed for pulses of duration $\tau:=0.5 \mu \mathrm{s}$. For a sound speed of $c_{s}=1.510^{3} \mathrm{~m} / \mathrm{s}$, this corresponds to a spatial scale of $0.75 \mathrm{~mm}$. In other words, the approximation (7) is valid for time scales larger than $0.5 \mu \mathrm{s}$, which corresponds to a physical resolution (typically chosen to be $\frac{1}{2} \lambda$ ) limited by $\lambda=0.75 \mathrm{~mm}$.

Real measurements correspond to sources of the form $\phi^{2}(t) * H(x) \delta_{0}(t)$, which are therefore convolved at the time scale $\tau$. This corresponds in the reconstruction of $H(x)$ to a blurring at the scale $\lambda$. As a consequence, details of $H(x)$ at the scale below $\frac{1}{2} \lambda$ cannot be reconstructed in a stable manner.

At any rate, we assume the first step in thermoacoustic tomography, namely the reconstruction of $H(x)$ from knowledge of pressure $p(t, x)$ on $\partial X$ done. It then remains to reconstruct $\sigma$ from knowledge of $H$.

\subsection{Inverse Scattering Problems with Internal Data}

We are now ready to present the inverse problems with internal data we consider in this paper. We recall that $u$ is modeled as the solution to (4). The incoming condition is imposed experimentally. We assume here that we can construct prescribed illuminations $u^{i}$ given as arbitrary superposition of plane waves $e^{i k \xi \cdot x}$ with $\xi \in \mathbb{S}^{2}$.

Traces $u_{\partial X}$ of solutions to (4) are dense in $H^{\frac{1}{2}}(\partial X)$ [15]. As a consequence, for each $g \in H^{\frac{1}{2}}(\partial X)$, we can find a sequence of illuminations $u^{i}$ such that the solution $u$ in the limit is the solution of

$$
\begin{array}{ll}
\Delta u+k^{2} u+i k \sigma(x) u=0, & X \\
u=g & \partial X,
\end{array}
$$

for a given boundary condition $g(x)$. The internal data are then of the form

$$
H(x)=\sigma(x)|u|^{2}(x) .
$$

It remains to find procedures that allow us to uniquely reconstruct $\sigma(x)$ from knowledge of $H(x)$ with a given illumination. We shall consider two different theories. The first one assumes a specific form of $g$ but allows us to reconstruct arbitrary (sufficiently smooth) $\sigma$ while the second one applies for general illuminations $g$ but only for small (though not necessarily smooth) functions $\sigma(x)$.

The above derivation can be generalized to the full system of Maxwell's equations. We then obtain the following inverse problem. Radiation is modeled by

$$
\begin{aligned}
& -\nabla \times \nabla \times E+k^{2} E+i k \sigma(x) E=0, \quad X \\
& \nu \times E=g \quad \partial X \text {. }
\end{aligned}
$$

In the vectorial case, the internal data, i.e., the amount of absorbed electromagnetic radiation, are then of the form

$$
H(x)=\sigma(x)|E|^{2}(x)
$$




\section{A general class of inverse problems with internal data}

The above problems are examples of a more general class we define as follows. Let $P(x, D)$ be an operator acting on functions defined in $\mathbb{C}^{m}$ for $m \in \mathbb{N}^{*}$ an integer and with values in the same space and let $B$ be a linear boundary operator defined in the same spaces. Consider the equation

$$
\begin{aligned}
P(x, D) u & =\sigma(x) u, & & x \in X \\
B u & =g, & & x \in \partial X .
\end{aligned}
$$

We assume that the above equation admits a unique weak solution in some Hilbert space $\mathcal{H}_{1}$ for sufficiently smooth illuminations $g(x)$ on $\partial X$.

For instance, $P$ could be the Helmholtz operator $i k^{-1}\left(\Delta+k^{2}\right)$ seen in the preceding section with $u \in \mathcal{H}_{1}:=H^{1}(X ; \mathbb{C})$ and $g \in H^{\frac{1}{2}}(\partial X ; \mathbb{C})$ with $B$ the trace operator on $\partial X$. Time -harmonic Maxwell's equations can be put in that framework with $m=n$ and

$$
P(x, D)=\frac{1}{i k}\left(\nabla \times \nabla \times-k^{2}\right) .
$$

We impose an additional constraint on $P(x, D)$ that the equation $P(x, D) u=f$ on $X$ with $B u=0$ on $\partial X$ admits a unique solution in $\mathcal{H}=L^{2}\left(X ; \mathbb{C}^{m}\right)$. For instance, $\mathcal{H}=L^{2}(X ; \mathbb{C})$ in the example seen in the preceding section in the scalar approximation provided that $k^{2}$ is not an eigenvalue of $-\Delta$ on $X$. For Maxwell's equations, the above constraint is satisfied so long as $k^{2}$ is not an internal eigenvalue of the Maxwell operator [8]. This is expressed by the existence of a constant $\alpha>0$ such that:

$$
(P(x, D) u, u)_{\mathcal{H}} \geq \alpha(u, u)_{\mathcal{H}} .
$$

Finally we assume that the conductivity $\sigma$ is bounded from above by a positive constant:

$$
0<\sigma(x) \leq \sigma_{M}(x) \quad \text { a.e. } x \in X
$$

We denote by $\Sigma_{M}$ the space of functions $\sigma(x)$ such that (15) holds. Measurements are then of the form $H(x)=\sigma(x)|u|^{2}$, where $|\cdot|$ is the Euclidean norm on $\mathbb{C}^{m}$. Then we have the following result.

Theorem 3.1 Let $\sigma_{j} \in \Sigma_{M}$ for $j=1,2$. Let $u_{j}$ be the solution to $P(x, D) u_{j}=\sigma_{j} u_{j}$ in $X$ with $B u_{j}=g$ on $\partial X$ for $j=1,2$. Define the internal data $H_{j}(x)=\sigma_{j}(x)\left|u_{j}(x)\right|^{2}$ on $X$.

Then for $\sigma_{M}$ sufficiently small so that $\sigma_{M}<\alpha$, we find that:

(i) [Uniqueness] If $H_{1}=H_{2}$ a.e. in $X$, then $\sigma_{1}(x)=\sigma_{2}(x)$ a.e. in $X$ where $H_{1}=H_{2}>0$.

(ii) [Stability] Moreover, we have the following stability estimate

$$
\left\|w_{1}\left(\sqrt{\sigma_{1}}-\sqrt{\sigma_{2}}\right)\right\|_{\mathcal{H}} \leq C\left\|w_{2}\left(\sqrt{H_{1}}-\sqrt{H_{2}}\right)\right\|_{\mathcal{H}},
$$

for some universal constant $C$ and for positive weights given by

$$
w_{1}^{2}(x)=\prod_{j=1,2} \frac{\left|u_{j}\right|}{\sqrt{\sigma}_{j}}(x), \quad w_{2}(x)=\frac{1}{\alpha-\sup _{x \in X} \sqrt{\sigma_{1} \sigma_{2}}} \max _{j=1,2} \frac{\sqrt{\sigma_{j}}}{\left|u_{j^{\prime}}\right|}(x)+\max _{j=1,2} \frac{1}{\sqrt{\sigma}_{j}}(x) .
$$


Here $j^{\prime}(j)=(2,1)$ for $j=(1,2)$.

The weights $w_{1}$ and $w_{2}$ are written here in terms of the conductivities $\sigma_{j}$ and the radiation solutions $u_{j}$. Under appropriate conditions on the solution $u_{j}$, these weights can be bounded above and below by explicit constants. In such circumstances, the above theorem thus shows that the reconstruction of $\sigma$ is Lipschitz-stable in $\mathcal{H}$ with respect to errors in the available data $H$.

Proof. Some straightforward algebra shows that

$$
P(x, D)\left(u_{1}-u_{2}\right)=\sqrt{\sigma_{1} \sigma_{2}}\left(\left|u_{2}\right| \hat{u}_{1}-\left|u_{1}\right| \hat{u}_{2}\right)+\left(\sqrt{H_{1}}-\sqrt{H_{2}}\right)\left(\frac{\sqrt{\sigma_{1}}}{\left|u_{1}\right|}-\frac{\sqrt{\sigma_{2}}}{\left|u_{2}\right|}\right) .
$$

Here we have defined $\hat{u}=\frac{u}{|u|}$. Although this does not constitute an equation for $u_{1}-u_{2}$, it turns out that

$$
|| u_{2}\left|\hat{u}_{1}-\right| u_{1}\left|\hat{u}_{2}\right|=\left|u_{2}-u_{1}\right|
$$

as can easily be verified. Our assumptions on $P(x, D)$ then imply that

$$
\left(\alpha-\sup _{x \in X} \sqrt{\sigma_{1} \sigma_{2}}\right)\left\|u_{1}-u_{2}\right\|_{\mathcal{H}}^{2} \leq\left(\left(\sqrt{H_{1}}-\sqrt{H_{2}}\right)\left(\frac{\sqrt{\sigma_{1}}}{\left|u_{1}\right|}-\frac{\sqrt{\sigma_{2}}}{\left|u_{2}\right|}\right), u_{1}-u_{2}\right)_{\mathcal{H}}
$$

and a corresponding bound for $u_{1}-u_{2}$ :

$$
\left(\alpha-\sup _{x \in X} \sqrt{\sigma_{1} \sigma_{2}}\right)\left\|u_{1}-u_{2}\right\|_{\mathcal{H}} \leq\left\|\left(\sqrt{H_{1}}-\sqrt{H_{2}}\right)\left(\frac{\sqrt{\sigma_{1}}}{\left|u_{1}\right|}-\frac{\sqrt{\sigma_{2}}}{\left|u_{2}\right|}\right)\right\|_{\mathcal{H}} .
$$

Now, we find that

$$
\left|u_{1}\right|-\left|u_{2}\right|=\frac{\sqrt{H_{1}}}{\sqrt{\sigma_{1}}}-\frac{\sqrt{H_{2}}}{\sqrt{\sigma_{2}}}=\left(H_{1} H_{2}\right)^{\frac{1}{4}}\left(\frac{1}{\sqrt{\sigma_{1}}}-\frac{1}{\sqrt{\sigma_{2}}}\right)+\left(H_{1}^{\frac{1}{4}}-H_{2}^{\frac{1}{4}}\right)\left(\frac{H_{1}^{\frac{1}{4}}}{\sqrt{\sigma_{1}}}-\frac{H_{2}^{\frac{1}{4}}}{\sqrt{\sigma_{2}}}\right) .
$$

Using $\left(H_{1}^{\frac{1}{4}}-H_{2}^{\frac{1}{4}}\right)\left(H_{1}^{\frac{1}{4}}+H_{2}^{\frac{1}{4}}\right)=\left(H_{1}^{\frac{1}{2}}-H_{2}^{\frac{1}{2}}\right)$ and ||$u_{1}|-| u_{2}|| \leq\left|u_{1}-u_{2}\right|$, we obtain (16).

Reconstructions for a simplified acousto-optics problem. In thermo-acoustics, the operator $P(x, D)$ considered in the preceding section is purely imaginary as can be seen in (13). In a simplified version of the acousto-optics problem considered in [3], it is interesting to look at the problem where $P(x, D)=\Delta$ and where the measurements are given by $H(x)=\sigma(x) u^{2}(x)$. Here, $u$ is thus the solution of the elliptic equation $(-\Delta+\sigma) u=0$ on $X$ with $u=g$ on $\partial X$. Assuming that $g$ is non-negative, which is the physically interesting case, we obtain that $|u|=u$ and hence

$$
\Delta\left(u_{1}-u_{2}\right)=\sqrt{\sigma_{1} \sigma_{2}}\left(u_{2}-u_{1}\right)+\left(\sqrt{H_{1}}-\sqrt{H_{2}}\right)\left(\frac{\sigma_{1}}{\sqrt{H_{1}}}-\frac{\sigma_{2}}{\sqrt{H_{2}}}\right) .
$$

Therefore, as soon as 0 is not an eigenvalue of $\Delta+\sqrt{\sigma_{1} \sigma_{2}}$, we obtain that $u_{1}=u_{2}$ and hence that $\sigma_{1}=\sigma_{2}$. In this situation, we do not need the constraint that $\sigma$ needs to be small. For instance, for $\sigma_{0}$ such that 0 is not an eigenvalue of $\Delta+\sigma_{0}$, we find that for 
$\sigma_{1}$ and $\sigma_{2}$ sufficiently close to $\sigma_{0}$, then $H_{1}=H_{2}$ implies that $\sigma_{1}=\sigma_{2}$ on the support of $H_{1}=H_{2}$. This particular situation, where a spectral equation arises for $u_{2}-u_{1}$, seems to be specific to the problem with $P(x, D)=\Delta$ and $u$ is a non-negative scalar quantity. Similarly, when $P(x, D)=-\Delta$, it was proved recently in [24] that the measurements uniquely and stably determine $\sigma$ in the Helmholtz equation $(\Delta+\sigma) u=0$.

In the setting of a thermo-acoustic problem, the theorem presented above is valid only when the $\sigma$ is sufficiently small. In the scalar case of thermo-acoustics, the smallness condition can be removed under additional assumptions on the illumination $g$.

\section{The scalar Helmholtz equation.}

Such illuminations are constructed using CGO solutions as in the inverse problem considered in [4], although the method of proof is somewhat different in the thermoacoustic context. Our proof here is based on showing that an appropriate functional of $\sigma$ admits a unique fixed point. This can be done when the conductivity $\sigma$ is sufficiently smooth. We assume that $\sigma \in H^{p}(X)$ for $p>\frac{n}{2}$ and construct

$$
q(x)=k^{2}+i k \sigma(x) \in H^{p}(X), \quad p>\frac{n}{2} .
$$

Moreover, $q(x)$ is the restriction to $X$ of the compactly supported function (still called q) $q \in H^{p}\left(\mathbb{R}^{n}\right)$. The extension is chosen so that [23, Chapter VI, Theorem 5]

$$
\left\|q_{\mid X}\right\|_{H^{p}(X)} \leq C\|q\|_{H^{p}\left(\mathbb{R}^{n}\right)}
$$

for some constant $C$ independent of $q$.

Then (8) is recast as

$$
\begin{aligned}
& \Delta u+q(x) u=0, \quad X \\
& u=g \quad \partial X \text {. }
\end{aligned}
$$

We recall that measurements are of the form $H(x)=\sigma(x)|u|^{2}(x)$.

We construct $g$ as the trace of the CGO solution of

$$
\Delta u+q u=0, \quad \mathbb{R}^{n},
$$

with $u=e^{\rho \cdot x}\left(1+\psi_{\rho}\right)$ with

$$
\rho \cdot \rho=0
$$

and $\psi_{\rho}$ solution in $L_{\delta}^{2}$ of the equation:

$$
\Delta \psi_{\rho}+2 \rho \cdot \nabla \psi_{\rho}=-q(x)\left(1+\psi_{\rho}\right), \quad \mathbb{R}^{n} .
$$

The data then take the form

$$
e^{-(\rho+\bar{\rho}) \cdot x} H(x)=\sigma(x)+\mathcal{H}[\sigma](x), \quad \mathcal{H}[\sigma](x)=\sigma(x)\left(\psi_{\rho}+\overline{\psi_{\rho}}+\psi_{\rho} \overline{\psi_{\rho}}\right) .
$$


It remains to show that $\mathcal{H}[\sigma](x)$ is a contraction, which is a sufficient condition for (24) to admit a unique solution. We do this in the space $Y=H^{\frac{n}{2}+\varepsilon}(X)$ where $n$ is spatial dimension. This is an algebra and we know that for $\sigma$ in that space, then the restriction of $\psi_{\rho}$ to $X$ is also in that space. Moreover $\rho \psi_{\rho}$ is bounded in that norm independent of $\rho$. The contraction property of $\mathcal{H}$ for $\rho$ sufficiently large stems from the contraction property of $\sigma \rightarrow \psi_{\rho}[\sigma]$ for $\rho$ sufficiently large. For the latter, we need to adapt the results proved in [4] to obtain Lipschitzness of $\psi_{\rho}$ with respect to $\sigma$ in the space $Y$ with its natural norm.

The Lipschitzness is obtained as follows. We introduce the spaces $H_{\delta}^{s}$ for $s \geq 0$ as the completion of $C_{0}^{\infty}\left(\mathbb{R}^{n}\right)$ with respect to the norm $\|\cdot\|_{H_{\delta}^{s}}$ defined as

$$
\|u\|_{H_{\delta}^{s}}=\left(\int_{\mathbb{R}^{n}}\langle x\rangle^{2 \delta}\left|(I-\Delta)^{\frac{s}{2}} u\right|^{2} d x\right)^{\frac{1}{2}} .
$$

Here $(I-\Delta)^{\frac{s}{2}} u$ is defined as the inverse Fourier transform of $\langle\xi\rangle^{s} \hat{u}(\xi)$, where $\hat{u}(\xi)$ is the Fourier transform of $u(x)$.

We define $Y=H^{p}(X)$ and $\mathcal{M}$ as the space of functions in $Y$ with norm bounded by a fixed $M>0$.

Lemma 4.1 Let $\psi$ be the solution of

$$
\Delta \psi+2 \rho \cdot \nabla \psi=-q(1+\psi)
$$

and $\tilde{\psi}$ be the solution of the same equation with $q$ replaced by $\tilde{q}$, where $\tilde{q}$ is defined as in (18) with $\sigma$ replaced by $\tilde{\sigma}$. We assume that $q$ and $\tilde{q}$ are in $\mathcal{M}$. Then there is a constant $C$ such that for all $\rho$ with $|\rho| \geq\left|\rho_{0}\right|$, we have

$$
\|\psi-\tilde{\psi}\|_{Y} \leq \frac{C}{|\rho|}\|\sigma-\tilde{\sigma}\|_{Y}
$$

Proof. We proceed as in [4] and write

$$
\psi=\sum_{j \geq 0} \psi_{j}
$$

with

$$
(\Delta+2 \rho \cdot \nabla) \psi_{j}=-q \psi_{j-1}, \quad j \geq 0
$$

with $\psi_{-1}=1$. This implies that

$$
(\Delta+2 \rho \cdot \nabla)\left(\psi_{j}-\tilde{\psi}_{j}\right)=-\left((q-\tilde{q}) \psi_{j-1}+\tilde{q}\left(\psi_{j-1}-\tilde{\psi}_{j-1}\right)\right)
$$

We recall the definition in [4] of the spaces $H_{\delta}^{s}$ for $s \geq 0$ as the completion of $C_{0}^{\infty}\left(\mathbb{R}^{n}\right)$ with respect to the norm $\|\cdot\|_{H_{\delta}^{s}}$ defined as

$$
\|u\|_{H_{\delta}^{s}}=\left(\int_{\mathbb{R}^{n}}\langle x\rangle^{2 \delta}\left|(I-\Delta)^{\frac{s}{2}} u\right|^{2} d x\right)^{\frac{1}{2}}, \quad\langle x\rangle=\left(1+|x|^{2}\right)^{\frac{1}{2}} .
$$


In the proof of [4, Proposition 3.1], it is shown that for some constant $C$ independent of $\rho$ for $|\rho|$ sufficiently large,

$$
\left\|\psi_{j}\right\|_{H_{\delta}^{s}} \leq C|\rho|^{-1}\|q\|_{H_{1}^{s}}\left\|\psi_{j-1}\right\|_{H_{\delta}^{s}}
$$

where by a slight abuse of notation, $\left\|\psi_{-1}\right\|_{H_{\delta}^{s}} \equiv 1$. We assume that $C|\rho|^{-1}\|q\|_{H_{1}^{s}}<1$ for $|\rho|$ sufficiently large so that the above series is summable in $j$. Let us still denote by $\psi_{j}$ the restriction of $\psi_{j}$ to $X$. Since $\langle x\rangle$ is bounded on $X$, we deduce from (19) that

$$
\left\|\psi_{j}\right\|_{Y} \leq C|\rho|^{-1}\|q\|_{Y}\left\|\psi_{j-1}\right\|_{Y}
$$

Upon defining $\varepsilon_{j}=\left\|\psi_{j}-\tilde{\psi}_{j}\right\|_{Y}$, we deduce from (31) and (29) that for some constant C,

$$
|\rho| \varepsilon_{j} \leq C\left\|\psi_{j-1}\right\|_{Y}\|q-\tilde{q}\|_{Y}+C M \varepsilon_{j-1},
$$

with $\varepsilon_{-1}=0$. Summing over $0 \leq j \leq J$ yields

$$
\sum_{j=0}^{J} \varepsilon_{j} \leq \frac{C}{|\rho|}\|q-\tilde{q}\|_{Y} \sum_{j=0}^{J}\left\|\psi_{j-1}\right\|_{Y}+\frac{C M}{|\rho|} \sum_{j=0}^{J-1} \varepsilon_{j}
$$

When $\rho$ is sufficiently large so that $\mathfrak{r}:=C M|\rho|^{-1}<1$, we obtain that

$$
\sum_{j \geq 0} \varepsilon_{j} \leq \frac{C\|q-\tilde{q}\|_{Y}}{(1-\mathfrak{r})|\rho|} \sum_{j \geq 0}\left\|\psi_{j-1}\right\|_{Y} \leq \frac{C^{\prime}}{(1-\mathfrak{r})|\rho|}\left(1+\|q\|_{Y}\right)\|q-\tilde{q}\|_{Y}
$$

This shows that

$$
\|\psi-\tilde{\psi}\|_{Y} \leq \sum_{j \geq 0}\|\psi-\tilde{\psi}\|_{Y} \leq \frac{C}{|\rho|}\|q-\tilde{q}\|_{Y} \leq \frac{C k}{|\rho|}\|\sigma-\tilde{\sigma}\|_{Y}
$$

This concludes the proof of the lemma.

From this, we obtain the

Corollary 4.2 Let $|\rho| \geq \rho_{0}$ for $\rho_{0}$ sufficiently large. Then there exists a constant $\mathfrak{r}<1$ such that

$$
\|\mathcal{H}[\sigma]-\mathcal{H}[\tilde{\sigma}]\|_{Y} \leq \mathfrak{r}\|\sigma-\tilde{\sigma}\|_{Y}
$$

Proof. We recall that

$$
\mathcal{H}[\sigma](x)=\sigma(x)\left(\psi_{\rho}+\overline{\psi_{\rho}}+\psi_{\rho} \overline{\psi_{\rho}}\right) .
$$

Since $Y$ is an algebra and $\sigma$ and $\psi_{\rho}$ are bounded in $Y$, we deduce from the preceding lemma the existence of a constant $C$ such that

$$
\|\mathcal{H}[\sigma]-\mathcal{H}[\tilde{\sigma}]\|_{Y} \leq \frac{C}{|\rho|}\|\sigma-\tilde{\sigma}\|_{Y}
$$

It remains to choose $|\rho|$ sufficiently large to show that $\mathcal{H}$ is a contraction from $Y$ to $Y$.

We thus deduce the reconstruction algorithm

$$
\sigma=\lim _{m \rightarrow \infty} \sigma_{m}, \quad \sigma_{0}=0, \quad \sigma_{m}(x)=e^{-(\rho+\bar{\rho}) \cdot x} H(x)-\mathcal{H}\left[\sigma_{m-1}\right](x), m \geq 1 .
$$


Quantitative Thermo-acoustics and related problems

4.1. Reconstructions for the inverse scattering problem

The above method allows us to uniquely reconstruct $\sigma$ provided that $g_{\rho}:=u_{\rho \mid \partial X}$ is chosen as a boundary illumination. Let now $g$ be close to $g_{\rho}$ and define

$$
(\Delta+q) u=0, \quad X, \quad u=g \quad \partial X
$$

We also define $\tilde{u}_{\rho}$ the CGO calculated with $\tilde{q}$ with trace $\tilde{g}_{\rho}$ on $\partial X$ as well as $\tilde{u}$ solution of

$$
(\Delta+\tilde{q}) \tilde{u}=0, \quad X, \quad \tilde{u}=g \quad \partial X .
$$

Then we find that

$$
(\Delta+q)\left(u-u_{\rho}\right)=0, \quad X, \quad u-u_{\rho}=g-g_{\rho} \quad \partial X
$$

as well as

$$
(\Delta+\tilde{q})\left(\tilde{u}-\tilde{u}_{\rho}\right)=0, \quad X, \quad \tilde{u}-\tilde{u}_{\rho}=g-\tilde{g}_{\rho} \quad \partial X .
$$

Let us define $Z=H^{p-\frac{1}{2}}(\partial X)$. We can show the following result

Theorem 4.3 Let $\rho \in \mathbb{C}^{n}$ be such that $|\rho|$ is sufficiently large and $\rho \cdot \rho=0$. Let $\sigma$ and $\tilde{\sigma}$ be functions in $\mathcal{M}$.

Let $g \in Z$ be a given illumination and $H(x)$ be the measurement given in (9) for $u$ solution of (8). Let $\tilde{H}(x)$ be the measurement constructed by replacing $\sigma$ by $\tilde{\sigma}$ in (9) and (8).

Then there is an open set of illuminations $g$ in $Z$ such that $H(x)=\tilde{H}(x)$ in $Y$ implies that $\sigma(x)=\tilde{\sigma}(x)$ in $Y$. Moreover, there exists a constant $C$ independent of $\sigma$ and $\tilde{\sigma}$ such that

$$
\|\sigma-\tilde{\sigma}\|_{Y} \leq C\|H-\tilde{H}\|_{Y}
$$

More precisely, we can write the reconstruction of $\sigma$ as finding the unique fixed point to the equation

$$
\sigma(x)=e^{-(\rho+\bar{\rho}) \cdot x} H(x)-\mathcal{H}_{g}[\sigma](x), \quad \text { in } Y .
$$

The functional $\mathcal{H}_{g}[\sigma]$ defined as

$$
\mathcal{H}_{g}[\sigma](x)=\sigma(x)\left(\psi_{g}(x)+\overline{\psi_{g}}(x)+\psi_{g}(x) \overline{\psi_{g}}(x)\right)
$$

is a contraction map for $g$ in the open set described above, where $\psi_{g}$ is defined as the solution to

$$
(\Delta+2 \rho \cdot \nabla) \psi_{g}=-q\left(1+\psi_{g}\right), \quad X, \quad \psi_{g}=e^{-\rho \cdot x} g-1 \quad \partial X
$$

We thus deduce the reconstruction algorithm

$$
\sigma=\lim _{m \rightarrow \infty} \sigma_{m}, \quad \sigma_{0}=0, \quad \sigma_{m}(x)=e^{-(\rho+\bar{\rho}) \cdot x} H(x)-\mathcal{H}_{g}\left[\sigma_{m-1}\right](x), m \geq 1
$$


Proof. We deduce from Lemma 4.1 and standard trace theorems that

$$
\left\|\psi_{\mid \partial X}-\tilde{\psi}_{\mid \partial X}\right\|_{Z} \leq \frac{C}{|\rho|}\|\sigma-\tilde{\sigma}\|_{Y}
$$

Let $g_{0}=e^{\rho \cdot x}\left(1+\psi_{\mid \partial X}\right)$ so that $\psi_{g_{0}}=\psi$. We have using Lemma 4.1 again and elliptic regularity of solutions to (37) that

$$
\left\|\psi_{g_{0}}-\tilde{\psi}_{g_{0}}\right\|_{Y} \leq\|\psi-\tilde{\psi}\|_{Y}+\left\|\tilde{\psi}-\tilde{\psi}_{g_{0}}\right\|_{Y} \leq\|\psi-\tilde{\psi}\|_{Y}+C\left\|\tilde{\psi}_{\partial X}-g_{0}\right\|_{Z} \leq \frac{C}{|\rho|}\|\sigma-\tilde{\sigma}\|_{Y} .
$$

Let now $g$ be close to $g_{0}$. Then we have

$$
\psi_{g}-\tilde{\psi}_{g}=\left[\left(\psi_{g}-\psi_{g_{0}}\right)-\left(\tilde{\psi}_{g}-\tilde{\psi}_{g_{0}}\right)\right]+\left(\psi_{g_{0}}-\tilde{\psi}_{g_{0}}\right) .
$$

It remains to analyze the term under square brackets. Define $\delta \psi=\psi_{g}-\psi_{g_{0}}$ with a similar notation for $\delta \tilde{\psi}$. Then we find that

$$
(\Delta+2 \rho \cdot \nabla+q)(\delta \psi-\delta \tilde{\psi})=(\tilde{q}-q) \delta \tilde{\psi}, \quad X, \quad \delta \psi-\delta \tilde{\psi}=0, \quad \partial X .
$$

Standard regularity results show that

$$
\|\left(\psi_{g}-\psi_{g_{0}}\right)-\left(\tilde{\psi}_{g}-\tilde{\psi}_{g_{0}}\left\|_{Y} \leq C\right\| \tilde{q}-q\left\|_{Y}\right\| g-g_{0} \|_{Z} .\right.
$$

As a consequence, we obtain that

$$
\left\|\psi_{g}-\tilde{\psi}_{g}\right\|_{Y} \leq C\left(\frac{1}{|\rho|}+\left\|e^{-\rho \cdot x}\left(g-g_{0}\right)\right\|_{Z}\right)\|\tilde{\sigma}-\sigma\|_{Y} .
$$

More generally, we find that

$$
\left\|\mathcal{H}_{g}[\sigma]-\mathcal{H}_{g}[\tilde{\sigma}]\right\|_{Y} \leq C\left(\frac{1}{|\rho|}+\left\|e^{-\rho \cdot x}\left(g-g_{0}\right)\right\|_{Z}\right)\|\tilde{\sigma}-\sigma\|_{Y}
$$

This shows that $\mathcal{H}_{g}[\sigma]-\mathcal{H}_{g}[\tilde{\sigma}]$ is a contraction for $|\rho|$ sufficiently large and $g$ in a sufficiently small open set around $g_{0}$. This concludes the proof of the theorem.

The above theorem provides a unique and stable reconstruction of $\sigma$ provided that the latter is sufficiently regular and provided that the illumination is sufficiently close to the CGO solutions that were constructed. The constraint on the illuminations is therefore not very explicit. The above method does not seem to directly generalize to the system of Maxwell's equations, where CGO solutions are more involved [27] and for which it is not clear that a contraction of the form (27) is available.

\section{Numerical experiments}

We now present some numerical simulations to verify the theory presented in the previous sections. To simplify the computation, we consider here only two-dimensional problems. In this section, the unit of length is centimeter $(\mathrm{cm})$ and that of the 
conductivity coefficient is Siemens per centimeter $\left(\mathrm{S} \mathrm{cm}^{-1}\right)$. The domain we take is the square $X=\left[\begin{array}{ll}0 & 2\end{array}\right] \times\left[\begin{array}{ll}0 & 2\end{array}\right]$. The permeability inside the domain is $\mu=50 \mu_{0}$, where $\mu_{0}$ is the vacuum permeability. The frequency of the problem is $3 \mathrm{GHz}$ so that the wavenumber $k \approx 2 \pi$ in all calculations. The conductivity $\sigma$ we reconstructed below is rescaled by $k c \mu$. These numbers are similar to those in [18]. Both the scalar Helmholtz equation and the vectorial Maxwell equation are solved with first order finite element method, implemented with the MATLAB software.

We implemented two different reconstruction algorithms. In the first algorithm, we reconstruct the unknowns by solving the least square minimization problem

$$
\sigma^{*}=\arg \min _{\sigma}\left\|H(\sigma)-H^{*}\right\|_{L^{2}(X)}^{2}+\gamma \mathcal{R}(\sigma) .
$$

where $\sigma$ is the unknown conductivity parameter to be reconstructed, $H^{*}$ is the interior data and $H(\sigma)$ is the model prediction given in (9) and (11), respectively, for the Helmholtz equation and the vectorial Maxwell equation. The regularization term $\mathcal{R}(\sigma)$ is selected as the Tikhonov regularization functional, the $L^{2}$ norm of $\nabla \sigma$. We solve the above least-square problem with a quasi-Newton type of iterative scheme implemented in $[20]$.

For the reconstruction in the Helmholtz case, we also implemented the iteration scheme (38) where we need to solve (37) at each iteration to find $\psi_{g}$. This equation is again discretized with a first order finite element scheme. The performance of the reconstruction scheme depends on the choice of the complex vector parameter $\rho$. The computational cost in the solution of (37) depends also on this parameter. For the problems we consider below, let us just mention that we selected $\rho=6 \zeta+6 i \zeta^{\perp}$ with $\zeta=(1,0), \zeta^{\perp}=(0,1)$. This $\rho$ makes (38) convergent while preventing the quantity $e^{-(\rho+\bar{\rho}) \cdot \mathbf{x}}$ from generating overflow. More details on the implementation this algorithm will be reported elsewhere.

Case 1. We first consider the reconstruction of the simple conductivity profile

$$
\sigma(\mathbf{x})= \begin{cases}0.6, & \mathbf{x} \in X_{1} \\ 0.4, & \mathbf{x} \in X_{2} \\ 0.2, & \mathbf{x} \in X \backslash\left(X_{1} \cup X_{2}\right)\end{cases}
$$

where $X_{1}=\{\mathbf{x}|| \mathbf{x}-(0.5,1) \mid \leq 0.2\}$ and $X_{2}$ is the rectangle $X_{2}=[1.31 .7] \times[0.51 .5]$. The true conductivity profile is shown in the top-left plot of Fig. 1. The synthetic data that we have constructed is shown on the top-right plot of the same figure. We performed four reconstructions. Two with noise-free data and two with noisy data that contain $10 \%$ multiplicative noise added by the algorithm $\tilde{H}=H *\left(1+\frac{\alpha}{100}\right.$ random $)$ with random a random field with values in $\left[\begin{array}{ll}-1 & 1\end{array}\right]$ and $\alpha$ the noise level. The reconstructions are given the second and third row of Fig. 1. The relative $L^{\infty}\left(L^{2}\right)$ error in the reconstructions with data for the two algorithms are $1.4 \%(0.1 \%)$ and $1.6 \%(0.1 \%)$, respectively, while those for the reconstructions with noisy data are $47.5 \%(3.1 \%)$ and $45.4 \%(3.6 \%)$ respectively. 

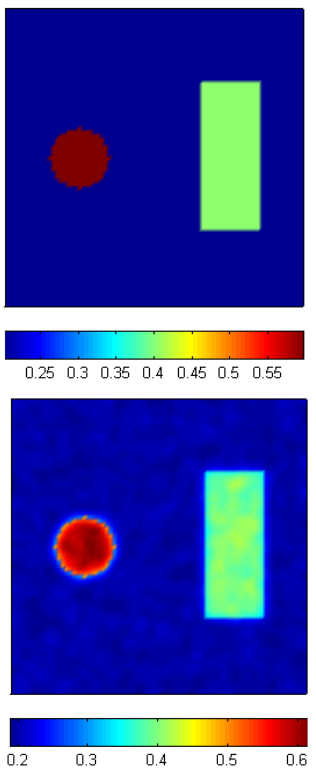
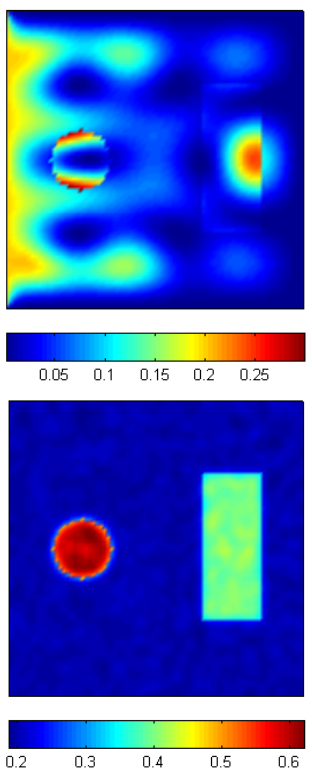
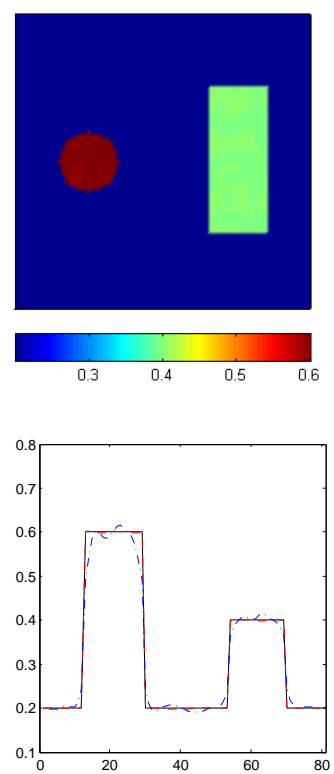
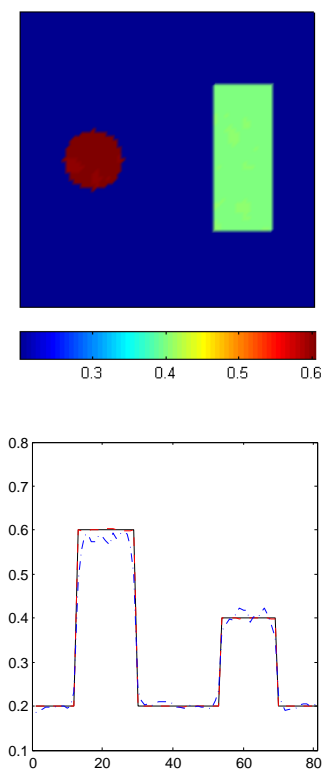

Figure 1. Reconstructions of an conductivity profile. From top left to bottom right: true conductivity, measurement $\sigma|u|^{2}$, reconstructed conductivity with first and second method with noise-free data, reconstructed conductivity with first and second method with noisy data, and the cross-sections of true (solid) and reconstructed (red dashed and blue dot-dashed) $\sigma$ along $y=1.0$

Case 2. We now attempt to reconstruct a more complicated conductivity profile, shown in the top-left plot of Fig. 2 from the Helmholtz equation. The conductivity is 0.2 in the background and 0.60 in the inclusion. The reconstructions are of similar quality as those in Fig. 1. The relative $L^{\infty}\left(L^{2}\right)$ error in the reconstructions with noise-free data for the two algorithms are $0.9 \%(0.1 \%)$ and $1.2 \%(0.1 \%)$, respectively, while those for the reconstructions with noisy data are $63.1 \%(5.8 \%)$ and $58.0 \%(5.1 \%)$, respectively.

Case 3. In the third numerical experiment, we consider reconstructions for the vectorial Maxwell equation. The conductivity profile considered here is the superposition of a homogeneous background and three disk inclusions. The conductivity is 0.2 in the background, 0.4, 0.6 and 0.8 in the three disks, respectively. As in the previous cases, the least-square algorithm seems to converge to a solution close to the true conductivity even when started relatively far away. It is, however, computationally preferable to put a constraint on the size of the conductivity coefficient so that it does not grow too large during quasi-Newton iteration. The reconstructions are given in Fig. 3. The relative $L^{\infty}\left(L^{2}\right)$ error in the reconstructions with noise-free data is $1.5 \%(0.2 \%)$ and that in the reconstructions with noisy data is $71.8 \%$ (3.3\%).

In general, we observe that the least-square algorithm works quite well. It usually converges in $6 \sim 10$ quasi-Newton iterations. The reconstruction scheme (38) yields very similar results to the least-square solutions. The speed of convergences, however, seems to be dependent on $\rho$. Also, the construction of the solution to (37) can be expensive 

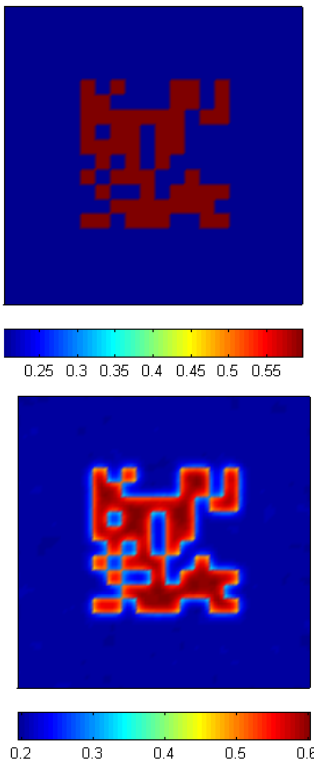
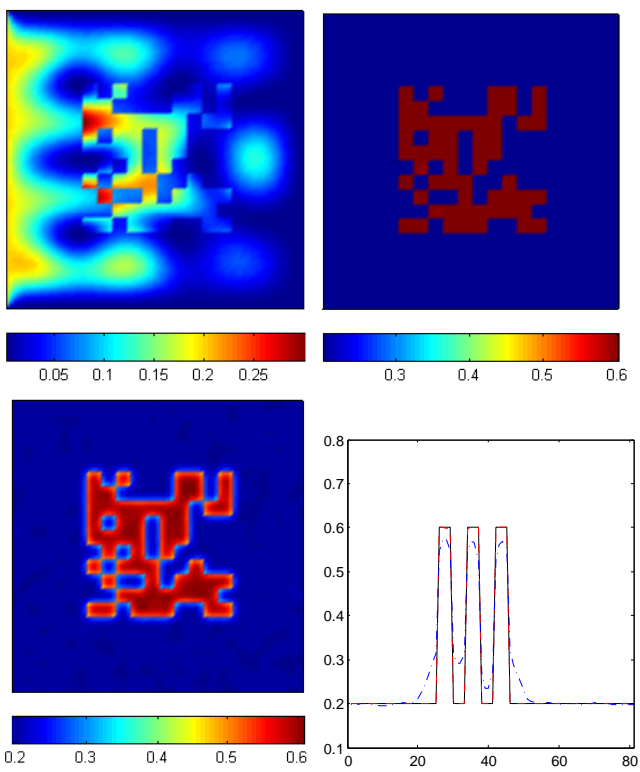
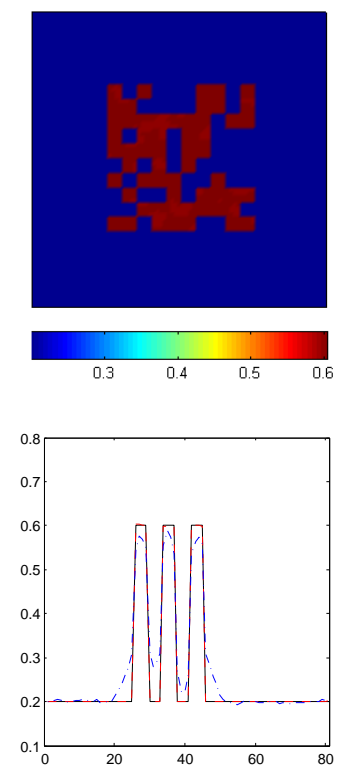

Figure 2. Reconstructions of an conductivity profile. From top left to bottom right: true conductivity, measurement $\sigma|u|^{2}$, reconstructed conductivity with first and second method with noise-free data, reconstructed conductivity with first and second method with noisy data, and the cross-sections of true (solid) and reconstructed (red dashed and blue dot-dashed) $\sigma$ along $y=1.0$

for $\rho$ with large amplitude.

\section{Acknowledgment}

GB was supported in part by NSF Grants DMS-0554097 and DMS-0804696. KR was supported in part by NSF Grant DMS-0914825. GU was partly supported by NSF, a Chancellor Professorship at UC Berkeley and a Senior Clay Award. TZ was partly supported by NSF grants DMS 0724808 and DMS 0758357.

\section{References}

[1] H. Ammari, E. Bossy, V. Jugnon, And H. Kang, Mathematical modelling in photo-acoustic imaging, to appear in SIAM Review, (2009).

[2] G. Bal, A. Jollivet, And V. Jugnon, Inverse transport theory of Photoacoustics, Inverse Problems, 26 (2010), p. 025011.

[3] G. Bal and J. C. Schotland, Inverse Scattering and Acousto-Optics Imaging, Phys. Rev. Letters, 104 (2010), p. 043902.

[4] G. Bal and G. Uhlmann, Inverse diffusion theory for photoacoustics, Inverse Problems, 26(8) (2010), p. 085010.

[5] B. T. Cox, S. R. Arridge, And P. C. Beard, Photoacoustic tomography with a limitedapterture planar sensor and a reverberant cavity, Inverse Problems, 23 (2007), pp. S95-S112.

[6] — Estimating chromophore distributions from multiwavelength photoacoustic images, J. Opt. Soc. Am. A, 26 (2009), pp. 443-455.

[7] B. T. Cox, J. G. Laufer, And P. C. BeArd, The challenges for quantitative photoacoustic imaging, Proc. of SPIE, 7177 (2009), p. 717713. 

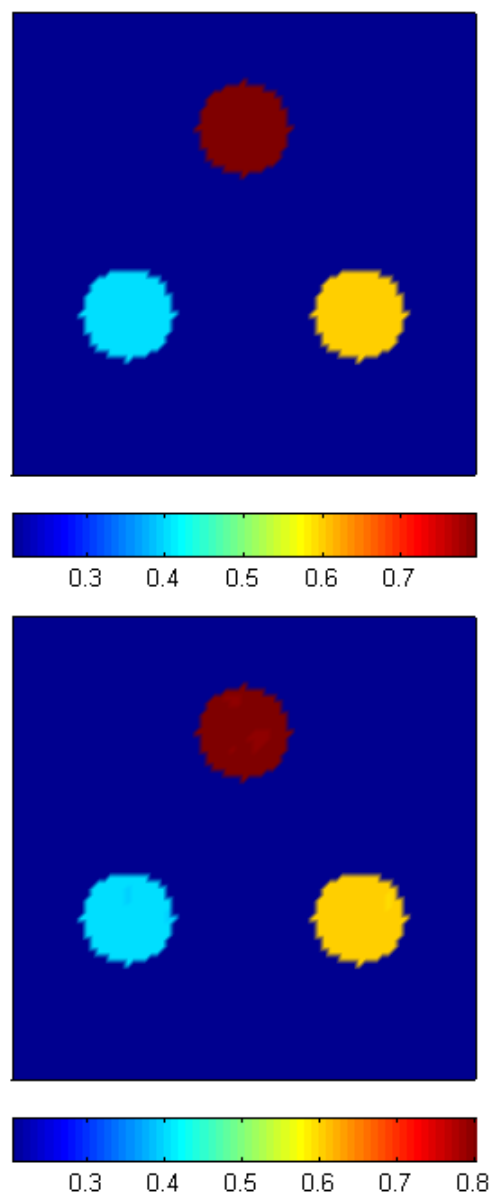
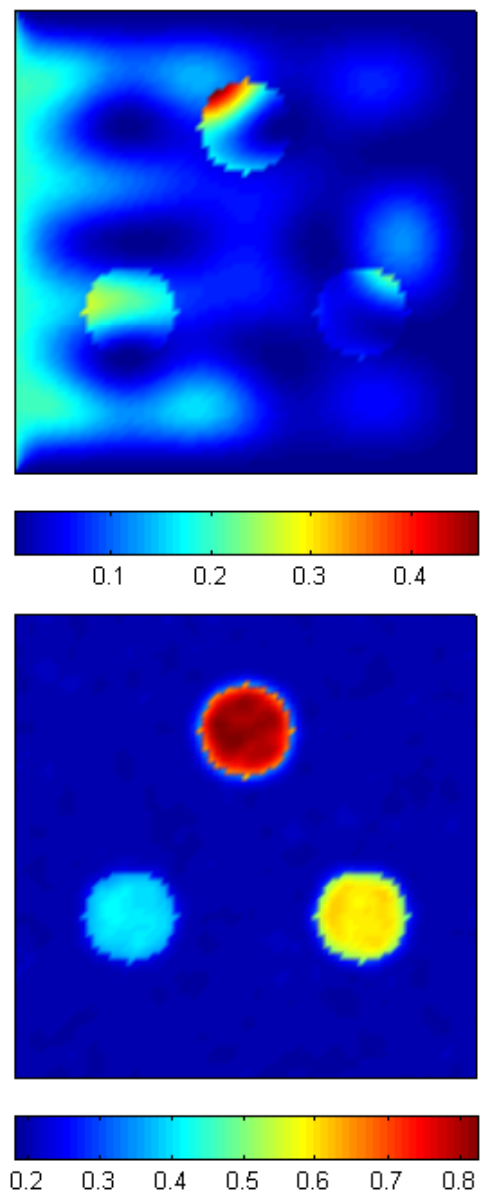

Figure 3. Reconstructions of an conductivity profile for the vectorial Maxwell equation. Top row: true conductivity and measurement $\sigma|E|^{2}$; Bottom row: conductivities reconstructed with noise-free data and noisy data.

[8] R. Dautray and J.-L. Lions, Mathematical Analysis and Numerical Methods for Science and Technology. Vol.3, Springer Verlag, Berlin, 1993.

[9] D. Finch And Rakesh., Recovering a function from its spherical mean values in two and three dimensions, in Photoacoustic imaging and spectroscopy L. H. Wang (Editor), CRC Press, (2009).

[10] S. K. Finch, D. Patch And RAKesh., Determining a function from its mean values over a family of spheres, SIAM J. Math. Anal., 35 (2004), pp. 1213-1240.

[11] A. R. Fisher, A. J. Schissler, And J. C. Schotland, Photoacoustic effect for multiply scattered light, Phys. Rev. E, 76 (2007), p. 036604.

[12] M. Haltmeier, O. Scherzer, P. Burgholzer, and G. Paltauf, Thermoacoustic computed tomography with large planar receivers, Inverse Problems, 20 (2004), pp. 1663-1673.

[13] M. Haltmeier, T. Schuster, and O. Scherzer, Filtered backprojection for thermoacoustic computed tomography in spherical geometry, Math. Methods Appl. Sci., 28 (2005), pp. 19191937.

[14] Y. Hristova, P. Kuchment, And L. Nguyen, Reconstruction and time reversal in thermoacoustic tomography in acoustically homogeneous and inhomogeneous media, Inverse Problems, 24 (2008), p. 055006.

[15] V. Isakov, Inverse Problems for Partial Differential Equations, Springer Verlag, New York, 1998.

[16] R. KowAR AND O. Scherzer, Photoacoustic imaging taking into account attenuation, in Mathematics and Algorithms in Tomography, vol. 18, Mathematisches Forschungsinstitut 
Oberwolfach, 2010, pp. 54-56.

[17] P. Kuchment and L. Kunyansky, Mathematics of thermoacoustic tomography, Euro. J. Appl. Math., 19 (2008), pp. 191-224.

[18] C. H. Li, M. Pramanik, G. Ku, And L. V. Wang, Image distortion in thermoacoustic tomography caused by microwave diffraction, Phys. Rev. E, 77 (2008), p. 031923.

[19] S. Patch And O. Scherzer, Photo- and thermo- acoustic imaging, Inverse Problems, 23 (2007), pp. S1-10.

[20] K. Ren, G. Bal, And A. H. Hielscher, Frequency domain optical tomography based on the equation of radiative transfer, SIAM J. Sci. Comput., 28 (2006), pp. 1463-1489.

[21] J. Ripoll AND V. NTZIAChristos, Quantitative point source photoacoustic inversion formulas for scattering and absorbing medium, Phys. Rev. E, 71 (2005), p. 031912.

[22] P. Stefanov and G. Uhlmann, Thermoacoustic tomography with variable sound speed, Inverse Problems, 25 (2009), p. 075011.

[23] E. Stein, Singular Integrals and Differentiability Properties of Functions, vol. 30 of Princeton Mathematical Series, Princeton University Press, Princeton, 1970.

[24] F. TRIKI, Uniqueness and stability for the inverse medium problem with internal data, Inverse Problems, 26 (2010), p. 095014.

[25] M. Xu And L. V. Wang, Photoacoustic imaging in biomedicine, Rev. Sci. Instr., 77 (2006), p. 041101.

[26] Y. Xu, L. Wang, P. Kuchment, and G. Ambartsoumian, Limited view thermoacoustic tomography, in Photoacoustic imaging and spectroscopy L. H. Wang (Editor), CRC Press, Ch. 6, (2009), pp. 61-73.

[27] T. Zhou, Reconstructing electromagnetic obstacles by the enclosure method, Inverse Probl. Imaging, 4(3) (2010), pp. 547-569. 